\title{
PHN List of Reviewers
}

The Editorial Board of Public Health Nutrition would like to thank the following for their contribution as peer reviewers in 2019:

Laura Aballay

Wafaa Abdelwahed

Abdul-Razak Abizari

Brenda Abu

Tania Aburto

Theo Adom

Aditika Agarwal

Kingsley Agho

Andres Agudelo-Suárez

Alicia Aguilar

Sixtus Aguree

Adelaide Agyepong

Faruk Ahmed

Selena Ahmed

Shakil Ahmed

Zaheer Ahmed

Maria Julia Ajejas Bazan

Mehtap Akçıl Ok

Shamima Akter

Harold Alderman

Ute Alexy

Asad Ali

Zainab Alimoradi

Margaret Allman-Farinelli

Naser Alsharairi

Jose Luis Alvarez Morán

Adeladza Amegah

Mohammad Reza Amiri

Dickson Amugsi

Costas Anastasiou

John Anderson

Elizabeth Anderson Steeves

Maria Andersson

Tatiana Andreyeva

Moira Donahue Angel

Jessica Appleton

Katherine Appleton

Jacqueline Araneda

Monica Arantxa Colchero

Joana Araujo

Gastón Ares Devincenzi

Danielle Arigo

Dachi Arikpo

Kokichi Arisawa
Amit Arora

Amelia Arria

Joanne Arsenault

Yuka Asada

Keiko Asakura

Mulusew Andualem Asemahagn

Waheed Mobolaji Ashagidigbi

Maria Alice Assis

Masanori Atsukawa

Amy Auchincloss

Dagfinn Aune

Bryn Austin

Philippe Autier

Rasmi Avula

Jennifer Ayton

Firas Sultan Azzeh

Ricardo Bado Pérez

Anne Bærug

Helen Bailey

Phillip Baker

Chrysa Bakoula

Aishat Bakre

Katie Balantekin

Kylie Ball

Valéria Baltar

Mahtab Bamji

Daniel Bandoni

Jonathan Banks

Jacob Bar

Larissa Baraldi

Janett Barbaresko

Alan Barclay

Mary Barker

Amy Barnes

Joaquin Barnoya

Sandhi Barreto

Katherine Barrett

Mikayla Barry

Otávio Bartalotti

Friederike Barthels

Ameena Batada

Malek Batal

Carolina Batis

Silvia Bauer
Lori Baugh Littlejohns

Ty Beal

Kathryn Beck

Carolyn Becker

Jacob Beckerman

Murat Bektas

Bethany Bell

Colin Bell

Bill Bellew

Katerina Belogianni

Ikram Benazizi

Yael Benn

Annemarie Bennett

Christie Bennett

Isabela Benseñor

Noelle Benzekri

Anselm Berde

Jacques Berger

Ingrid Bergstrom

James Berkley

Seth Berkowitz

Angela Bermudez-Millam

Antonio Bernabe-Ortiz

Jennifer Bernal

Greyce Bernardo

Jodi Bernstein

Ilana Bezerra

Ingrid Bezerra

Zulfiqar Bhutta

Daniel Bia

Sander Biesbroek

Natassja Billich

Alice Binder

Nancy Birungi

Ghose Bishwajit

Maureen M Black

Miranda Blake

Ewa Błaszczyk-Bębenek

Sara Bleich

Jonathan Blitstein

Lynne Boddy

Tara Boelsen-Robinson

Leonie Bogl

Sharmilah Booley 
Alison Booth

Camila Borges

Negussie Boti

Jeremie Botton

Vasiliki Bountziouka

Deborah Bowen

Erick Boy

Sinead Boylan

Emma Boyland

Loretta Brabin

Tilman Brand

Anne Lise Brantsæter

Francesca Bravi

Issy Bray

Helmut Breitmeier

Ronette Briefel

Andre Briend

Flávia dos Santos Brito

Christine Brombach

Louise Brough

Andrew Brown

Callie Brown

Heather Brown

Nick Brown

Debra Brucker

Meg Bruening

Angela Bruns

Andrea Buchholz

Opal Buchthal

Christina Buckton

Rhodi Bullock

Irena Buric

Michael Burke

Katherine Burt

Melissa Burton

Lucy Butcher

Judith Buttriss

Julie Byles

Karen Byrd

Rebecca Byrne

Anna C. Queiroz de Medeiros

Philip Calder

Ryan Calder

Julia Caldwell

Érika Camargo

Adrian Cameron

Aurore Camier

Elizabeth Campbell

Rebecca Campbell

Judy Canahuati

Daniela Canella

Helena Canhão

Jonathan Cantor

Raquel Canuto

Simon Capewell

Martin Caraher
Marly Cardoso

Júlia Cardoso Carraro

Rachel Carey

Julia Carins

Fiorella Carlos Chavez

Elena Carrillo Álvarez

Kathryn Carroll

JoAnn Carson

Maria Antonieta Carvalhaes

Carmen Casanovas

Andrea Cassidy-Bushrow

Gemma Castaño-Vinyals

Jessica Castonguay

Arachu Castro

Ines Rugani Castro

Bess Caswell

Laura Caulfield

Carlos Celis-Morales

Ana Carla Cepeda López

Suman Chakrabarti

Queenie Chan

Ruth Chan

Sheila Chanani

Hsing-Yi Chang

Yunhee Chang

Sarah Chantler

Angeline Chatelan

Rahul Chatterjee

Abhishek Chaudhary

Jorge Chavarro

Kee Chee Cheong

Bo Chen

Yu-ming Chen

Lan Cheng

Joelaine Chetty

Ching-Lung Cheung

Pheak Chhoun

Ai-Ru Chia

Katelyn Chiang

Mariana Chilton

Kik-Yong Chin

Esnat Chirwa

Eunyoung Cho

Gwen Chodur

Cláudia Choma

Nuzhat Choudhury

Benjamin Chrisinger

Meaghan Christian

Mary Christoph

Nopphanath Chumpathat

Ock Chun

Sochung Chung

Arrigo Cicero

Nick Clark

Natasha Clarke

Rafael Claro
Christelle Clary

Julie Cliff

Jennifer Cohen

Juliana Cohen

Carlos Coimbra Jr.

Tatiana Collese

Uriyoán Colón-Ramos

Lidia Compean-Ortiz

Dominique Condo

Annalijn Conklin

Tamlin Conner

Zach Conrad

Brian Cook

Marcia Cooper

Teresa Correa

Maria Correa-Rodriguez

Daniel Corsi

Bruna Costa

Maria Helena Costa

David Cox

Sandra Crispim

Pauline Croll

Sarah Crozier

Cristobal Cuadrado

Katherine Cullerton

Kenda Cunningham

Leslie Cunningham-Sabo

Cintia Curioni

Cynthia Curl

María Curutchet

Sebastien Czernichow

Heather D'Angelo

Luciana da Silva

Sara da Silva

Gloria da Veiga

Laura D'Addezio

Jai Dadhich

Madeleine Daepp

Zhaoli Dai

Lisa Daniels

Ina Danquah

Pauline Darling

Nicole Darmon

Ian Darnton-Hill

Amelia Darrouzet-Nardi

Saeed Dastgiri

Ashlesha Datar

Ian Davies

Jaimie Davis

Sally Davis

Karen Davison

Alan de Brauw

Gert-Jan de Bruijn

Maria Beatriz de Castro

Giovanni de Gaetano

Ros de Groot 
Blandine De Lauzon-Guillain Carlos de Mestral Milena de Moraes Amanda de Moura Souza Alessandro de Oliveira David de Oliveira Jamie de Seymour Marian de van der Schueren Corrado De Vito Luanne DeChristopher

Andrea Deierlein

Louise Dekker

Corinne Delamaire

Tessa Delaney

Miguel Delgado-Rodriguez

Hélène Delisle

Diane DellaValle

Treena Delormier

Zewditu Demissie

Nicole den Braver

Edgar Denova-Gutierrez

Katarzyna Dereń

Kristine Deroover

Dipti Dev

Jill Dever

Brecht Devleesschauwer

Robin DeWeese

Muhammad Dhansay

Jaapna Dhillon

Preeti Dhuria

Jennifer Di Noia

Aly Diana

Kacie Dickinson

Albert Do

Joannie Dobbs

Marcela Dofkova

Radhouene Doggui

David Doledec

Semíramis Domene

Shannon Donofry

Marie-Claude Dop

Sandra dos Santos

Mariska Dötsch-Klerk

Ian Dranton-Hill

Catherine Draper

Roisin Drysdale

Anna D'Souza

Audrée-Anne Dumas

Elizabeth Dunford

Anne Dunlop

Thomas Dunn

Ana Clara Duran

Solange Durao

Laura Dwyer

Creswell Eastman

Soraiya Ebrahimpour-koujan
Christine Edwards

Grace Egeland

Ans Eilander

Marla Eisenberg

Abdel Hamid El Bilbeisi

Karin Eli

Jordan Ellis

Sara Elnakib

Ahmed El-Sohemy

Pauline Emmett

Jennifer Emond

Ulrika Enemark

Emily Engelhard

Rachel Engler-Stringer

Linda Englund-Ögge

John Erdman Jr.

Nicole Erler

Iris Erlund

Emma Esdaile

Ahmad Ali Eslami

Charlotte Evans

Whitney Evans

Alexa Evenson

Alice Fabbri

Mieke Faber

Andrée-Anne Fafard St-Germain

Shiva Faghih

Nicole Fakhoury-Sayegh

Samaneh Farsijani

Sohani Fatehin

Flavia Fayet-Moore

Termeh Feinberg

Megan Ferguson

Ana Fernandes

Rômulo Araújo Fernandes

Maria Luz Fernandez

Juan Fernández-Alvira

Erin Ferranti

Fabrícia Ferreira

Daniele Mendonça Ferreira He

Giovanna Fiates

Giovanna M. R. Fiates

Barbara Fielding

Roger Figueroa

Kathryn Fiorella

Flavia Fioruci

Florian Fischer

Bridget C. Foley

Hannah Forde

Maryah Fram

Deborah Frank

David Frankenfield

Marco Franze

Darcy Freedman

Wilma Freire Zaldumbide

Heinz Freisling
Edward Frongillo Jr.

Takeo Fujiwara

Teresa Fung

Robert Fungo

Lebo Gafane-Matemane

Carlos Andres Gallegos Riofrio

Sina Gallo

Luiz A. C. Galvao

Wan Ying Gan

Vanessa Garcia-Larsen

Greg Garrett

Kayleigh Garthwaite

Pamela Gaskin

Giovanna Gatica-Domínguez

Jewel Gausman

Rozenn Gazan

Aulo Gelli

Cindy George

Elena George

Andreas Georgiadis

Mariette Gerber

Majid Ghayour-Mobarhan

Madhumita (Bonnie) Ghosh-

Dastidar

Suparna Ghosh-Jerath

Heather Gibbs

Simone Gibson

Selma Gicevic

Catherine Gichunge

Tiffany Gill

Jason Gilliland

Gary Ginsberg

Edward Giovannucci

Zemichael Gizaw

Dominika Głąbska

Marci Gluck

Kate Godden

Louise Goff

Moria Golan

Christopher Golden

Kate Golden

Sarah Gollust

Caroline Gomes

Silvia Gonzalez

Catalina González Hidalgo

Sandra Gonzalez Palacios

David Gonzalez-Chica

Laura González-Zapata

Bartira Gorgulho

Kathleen Gorman

Steven Gortmaker

Wendi Gosliner

Igor Grabovac

Sabrina Granheim

Antoneta Granic

William Grant 
Andrew Gray

Matthew Graziose

Amanda Grech

Sarah Green

Paola Gremigni

Carley Grimes

Louise Grimmer

Christa Grobler

Ida Gronborg

Sareen Gropper

Giuseppe Grosso

Anna Grummon

Aihua Gu

Vivienne Guan

Muriel Gubert

Enza Gucciardi

Carlos Guerrero-López

Craig Gundersen

Erdoğan Güneş

Yuming Guo

Adyya Gupta

Piyush Gupta

Ricardo Gurgel

Joanne Guthrie

Esperanza Gutierrez- Redomero

Josephine Gwynn

Daniel Gyamfi

Razak M. Gyasi

Mohammed Habibullah-AlMamun

Rebecca Hagedorn

Luc Hagenaars

Majid Hajifaraji

K. Michael Hambidge

Meagan Hanbury

Joyanna Hansen

Marjolein Harbers

Janetta Harbron

Kassandra Harding

Annie Hardison-Moody

Monica Hargraves

Margaret Hargreaves

Lisa Harnack

Neil Harris

Terryl Hartman

Anna Harton

Kenneth Harttgen

Irsan Hasan

Rebecca Hasdell

Nancy Haselow

Kawther Hashem

Danielle Haslam

Neva Hassanein

Habiba Hassan-Wassef

Nihal Hatipoglu

Benjamin Hawkins
Irana Hawkins

Nicola Hawley

Emma Haycraft

Lindsey Haynes-Maslow

Feng He

Jincai He

Yuan He

Amelie Hecht

Christina Hecht

William Heerman

Azita Hekmatdoost

Jaimie Hemsworth

Sheryl Hendriks

Florian Herbolsheimer

Anna Herforth

Dominica Hernandez

Mauricio Hernández-Fernandez

Cristina Herrera

Julie Hess

Marion Hetherington

Ghazaleh Heydarirad

Akihisa Hidaka

Deana Hildebrand

Alyson Hill

Jennie Hill

Jillian Hill

Tisa Hill

Elisabet Hillesund

Claudia Hitaj

Allison Hodge

Doroteia Höfelmann

Karen Hofman

Adrian Hoffmann

Emily Hohman

Michael Holick

Clare Holley

Bruce Hollis

Anna Holzscheiter

Laura C. Hopkins

Sinead Hopkins

Graham Horgan

Margrethe Horlyck-Romanovsky

Paula Horta

Farhad Hosseinpanah

Mohammad Javad HosseinzadehAttar

M. Hotta

Serena Houghton

Amber Hromi-Fiedler

Adela Hruby

Dongsheng $\mathrm{Hu}$

Mark Huffman

Clare Hughes

Roger Hughes

Paul Hulshof

Sandra Hunziker
Oliver Huse

Ena Huseinovic

Lieven Huybregts

Taisun Hyun

Scott Ickes

Iris Iglesia

Ezinne Igwe

Maj-Britt Inhulsen

Gabriela Interlenghi

Kazuko Ishikawa-Takata

M Mofizul Islam

Leila Itani

Suvi Itkonen

Kia Nöhr Iversen

T Iwahori

Masanori Iwasaki

Dylan Jackson

Enrique Jacoby

Paul Jacques

Tina Jafari

Lisa Jahns

Patricia Jaime

Erica Jansen

Megan Jarman

Renuka Jayatissa

Susan Jebb

Tamryn Jenkings

Katharine Jenner

Melissa Jensen

Pierre Jesus

Mengmeng Ji

Peng Jia

Hua Jiang

William Joe

William Johnson

Carol Johnston

Lamis Jomaa

Amanda Jones

Rebecca Jones

Rachel Jones

Chantal Julia

Niina Kaartinen

Enamul Kabir

Zubair Kabir

Gilberto Kac

Hasibe Kadıoğlu

Lucia Kaiser

Yuya Kakutani

Alexander Kalimbira

Louiza Kalokairinou

Shubhada Kanani

Hee-Taik Kang

Yunhee Kang

Deksha Kapoor

Tilakavati Karupaiah

Christina Kasprzak 
PHN List of Reviewers

Suna Kassier

Satvinder Kaur

Justine Kavle

Mike Keesman

Michelle Kegler

Mojtaba Keikha

Amelie Keller

Heather Keller

Jane Kellett

Matthew Kelly

Lauren Kennedy

Erica Kenney

Monique Potvin Kent

Jennifer Keogh

Marko Kerac

Mathilde Kersting

Saman Khalesi

Hina Khalid

Mohammad Khalili

Asad Khan

Hafiz Khan

Jahidur Khan

Md Khan

Shweta Khandelwal

Neha Khandpur

Anna Kharmats

Muhammad Khattak

Monica Kidd

Hyunju Kim

Jihye Kim

Kirang Kim

Mikyung Kim

Judith Kimiwe

Jin Kim-Mozeleski

Christian King

Lesley King

Sara Kirk

Sharon Kirkpatrick

Line Kleinebreil

Jacob Klerman

Linda Knol

Satomi Kobayashi

Eda Koksal

Jane Kolodinsky

Ourania Kolokotroni

Patrick Kolsteren

Joel Komakech

Keiko Kondo

Liisa Korkalo

Jana Korner

Monica Kothari

Albert Koulman

Małgorzata Kowal

Tatsuya Koyama

Slawomir Koziel

Sibylle Kranz
Michael Krawinkel

Maria F Kroker-Lobos

Jaclyn Kropp

Marie Kuczmarski

Tilman Kühn

Gunter Kuhnle

Archana Kujur

Kissa Kulwa

Archana Kumar

Chiza Kumwenda

Sarka Kunzova

Tony Kuo

Hebah Kutbi

Keisuke Kuwahara

Naa Oyo Kwate

Chris Kypridemos

Carlo La Vecchia

Ronald Labonte

Allison Lacko

Linnea Laestadius

Eamon Laird

Benjamin Chih Chiang Lam

Kelly Lambert

Hannah G. Lane

Tara LaRowe

Nicole Larson

Anna Lartey

Margaret Lawler

Mark Lawrence

Jean-Charles Leblanc

Hye-Ja Lee

Jane Lee

Patricia Lee

Sang-Ah Lee

Seung-Yeon Lee

Yongjae Lee

Sophie Lefèvre-Arbogast

Reetta Lehto

Kathleen Lenk

Marleen Lentjes

Karimen León-Flández

May May Leung

Carol Levin

Susan Levin

Elyse Levine

Kaigang Li

Li-ming Li

Sherly Li

Tongzhe Li

Wenjie Li

Yanping Li

Jessica Lieffers

Djin Gie Liem

Nanna Lien

Angela Liese

Mara Lima de Cnop
Panita Limpawattana

Wei-Quan Lin

Wei-Ting Lin

Rebecca Lindberg

Kristina Lindvall

Steven Lipshultz

Cosima Lisi

Betsy List

Junxiu Liu

Pablo Lizana Arce

Megan Lloyd

Brian Lo

Lindsey Locks

Antje Löffler

Caomhan Logue

Carl Lombard

Martani Lombard

Christopher Long

Michael W. Long

Marianne Lonnebotn

Aline Lopes

Carla Lopes

Nanette Lopez

Nancy López-Olmedo

Amy Lovell

Sean Lucan

Daniela Lucini

Janet Lydecker

Meghan Lynch

Leslie Lytle

Guansheng Ma

Jianto Ma

Xiaonan $\mathrm{Ma}$

Lawrence Mabasa

Lesley Macaskill

Priscila Pereira Machado

Una MacIntyre

Sally Mackay

Joreintje Mackenbach

Peter Madril

Catherine Mah

Trias Mahmudiono

Michele Malaguarnera

Linda Malan

Kimberley Mallan

Shakira Mandal

Reed Mangels

Mercy Manyema

Franca Marangoni

Sri Marliyati

Iva Marques-Lopes

Pedro Marques-Vidal

Bernadette Marriott

M. D. Marrodan

Corby Martin

Stephanie Martin 
Euridice Martinez Steele José M Martinez-Carrión Ana Martinez-Donate Judith Martin-Fernandez Diane Martins Marcia Martins Deborah Martone Miquel Martorell Segametsi Ditshebo Maruapula Wolfgang Marx Gertraud Maskarinec

Travis Masterson

Marco Mastroeni

Salvatore Andrea Mastrolia

Brittany Matheson

Kaaren Mathias

Susana Matias

Maria Gabriela Matias de Pinho

Mai Matsumoto

Mika Matsuzaki

Andrea Maugeri

Mohsen Mazidi

Blessing Mberu

Amanda McClain

James McClung

Judith McCool

Keri McCrickerd

Jessica L. McCurley

Pearl McElfish

Melissa McInerney

Lynn McIntyre

Patrick McLaughlin

Amy McLennan

Taylor McLinden

Maria Mclnerney

Emma McMahon

Pamela McMahon

Fernanda Mediano Stoltze

Sarah Meiklejohn

Jelena Meinilä

Aamir Memon

Marcela Mendes

Amy Mendham

Yu Meng

Caitlin Merlo

Amaka Metu

Alexandre Meybeck

Nanna Meyer

Melissa Mialon

Laura Miccoli

Agnieszka Micek

Juan Mielgo Ayuso

Seema Mihrshahi

José Mill

Daniel Miller

Jon Miller
Joshua Miller

Laurie Miller

D. Joe Millward

Jungwon Min

Leia Minaker

Maryam Miraghajani

Andreia Miranda

Parvin Mirmiran

Lorenza Mistura

Jonathan Mitchell

Kristin Mmari

Daniel Mochon

Noushin Mohammadifard

Viswanathan Mohan

Sumarni Mohd Ghazali

Zalilah Mohd Shariff

Masuda Mohsena

Marc Molendijk

Maria Del Carmen Molina

Rafael Monge Rojas

Ali Montazeri

Carlos Monteiro

Luana Monteiro

Marj Moodie

Latetia Moore

Ghobad Moradi

Mercedes Mora-Plazas

Mariana Isabel Valdés Moreno

Anna Morgan

Emily Morgan

Taylor Morris

Joanna Morrison

Jasper Most

Farideh Mostafavi

Irene Motta

Paula Moynihan

Rebecca Mozaffarian

Megan Mueller

Angela Mulligan

Christine Mulligan

Janet Mullins

Hilda Mulrooney

Rebekka Mumm

Kentaro Murakami

Jane Murphy

Mary Murphy

Aviva Musicus

Siti Muslimatun

Mark Myatt

Jannicke Myhre

Muzi Na

Mahdi Najafi

Harunobu Nakamura

Mieko Nakamura

Sorrel Namaste

Nazli Namazi
Ihab Naser

Androniki Naska

Lara Nassreddine

Nicole Nathan

Eva Maria Navarrete-Muñoz

Anderson Navarro

Rodolfo Nayga, Jr.

Tom Ndanu

Bruce Neal

Elizabeth Neale

Cindy Needham

Roni Neff

Etienne Nel

Michael Nelson

Marion Nestle

Judith Neter

Merryn Netting

Lynnette Neufeld

Clara Neves

Paulo Augusto Neves

Shu Wen Ng

Giang Nguyen

Theresa Nicklas

Jeff Niederdeppe

David Nieman

Claudia Nieto

F. Javier Nieto

Jeri Nieves

Annie Nigra

Roy Nilsen

Nobuo Nishi

Ramadhani Noor

Astrid Nooyens

Norazmir Md. Nor

Jennifer Norman

Tom Norris

Venetia Notara

Ute Nöthlings

Dariusz Nowak

Katerina Ntourou

Bruno Pereira Nunes

Ali Nurshad

Christina Nyhus

Sophie Ochola

Marga Ocké

Rebecca O'Connell

Sydney O'Connor

Mary Odum

Feyisayo Odunitan-Wayas

Felix Ogbo

Cynthia Ogden

Jeffery O'Hara

Megan O'Hearn

Punam Ohri-Vachaspati

Akira Okayama

Kufre Okop 
PHN List of Reviewers

Nagako Okuda

Wilna Oldewage-Theron

Andreia Oliveira

Julicristie Oliveira

Mayara Oliveira

Brittney Oliver

Rocio Olmedo-Requena

Rose Omari

Shu Hwa Ong

Stephen Onufrak

Rose Opiyo Okoyo

Ch Orces

Nicole O'Reilly

Michael Orlich

Colin Orr

Ana Ortega-Avila

Hibbah Osei-Kwasi

Alberto Rubén Osella

Hanife Özçelik

Helena Pachon

Mark Pachucki

Patricia Padilha

Sallie Page-Goertz

Hee Young Paik

J. M. Paik

Cristina Palacios

Lara Palmeira

Debbie Palmer

Mustafa Pamuk

Demosthenes Panagiotakos

Pallavi Panth

Angeliki Papadaki

Nikolaos Papanas

Yanni Papanikolaou

Keren Papier

Andrew Papworth

Sherly Parackal

Niyati Parekh

Songyi Park

Yong-Moon Park

Kyong Park

Whadi-ah Parker

Christine Parr

Diana Parra

Julio Parra F

Allison Parsons

Stephanie Partridge

Chloe Patel

Euan Paterson

Amanda Patterson

Joanne G Patterson

Julie Patterson

Kaitlin Patterson

Elise Pauzé

Elizabeth Pearce

Lilia Pedraza
Tatiana Pedroso de Paula

Nasheeta Peer

David Pell

Gretel Pelto

Mary Penny

Rosangela Pereira

Pablo Pereira

Catherine Pereira

Luis Pereira da Silva

Marcos Pereira-Santos

Carolina Pérez Ferrer

Federico Pérez-Cueto

Rafael Pérez-Escamilla

Mario Pérez-Zepeda

Susan Persky

Megan Pesch

Milene Pessoa

Kristina Petersen

Paul Petraro

Nick Petrunoff

Mark Petticrew

Simone Pettigrew

Emily A. Phillips

Greg Pierce

Silvia Pinhao Cruz

Erin Pitt

Maartje Poelman

Kalpagam Polasa

Jorge Polonia

Evangelos Polychronopoulos

Jennifer Pomeranz

Jennifer Pooler

Judi Porter

L. Kirsty Pourshahidi

Bronwen Powell

Katie Powell

Thomas Power

Adriyan Pramono

Igor Pravst

Melissa Prescott

Eduardo Pretell

Agatha Previdelli

Joseph Price

Yasmine Probst

Paolo Prosperi

Veronique Provencher

Rachel Prowse

Katarzyna Przybylowicz

Paolo Emilio Puddu

Claire Pulker

Juliet Pullar

Mohammad Habibullah Pulok

Seema Puri

Matin Qaim

Susan Raatz

Cynthia Radnitz
Kalyani Raghunathan

Berna Rahi

Andaleeb Rahman

Kim Raine

Paiva Rajala-Schultz

Janani Rajbhandari-Thapa

PuruShothama Rakesh

Hasina Rakotomanana

Divya Ramachandran

Amutha Ramadas

Alanderson Ramalho

Maria Jose Ramirez Luzuriaga

Naila Ramji

Janis Randall Simpson

Anna Rangan

Natalie Rangelov

Audrey Rankin

Neha Rathi

Fernanda Rauber

Jofrey Raymond

Jill Reedy

Erica Reeve

Sue Reeves

Aaron Reeves

David Rehkopf

Colin Rehm

Julia Reichenberger

Marla Reicks

Ian Reid

Kirsten Rennie

Cesar Revoredo Giha

Marcela Reyes

Renee Reynolds

Cecília Cláudia Ribeiro

Roberta Luksevicius Rica

Nadine Richter

Ronit Ridberg

Natascia Rinaldo

Sofia Rincon-Gallardo

Renata Ristic

Lorence Ritchie

Rene Rizzoli

Éric Robitaille

Brenda Robles

Shannon Robson

Paula Robson

Romana Roccaldo

Paulo Rodrigues

Luis Rodriguez

Alison Rogers

Sabine Rohrmann

Kaitlin Roke

Rimante Ronto

Donald Rose

Richard Rosenkranz

Jorge Rosenthal 
Asher Rosinger

Laura Rossi

Daniel Roth

Anne M. Roubal

Emily Rousham

Erin Ruel

Emma Ruiz

Miguel Ruiz-Canela

Iean Russell

Femke Rutters

Anna Saba

Gary Sacks

Mehdi Sadeghian

Richard Sadler

Emma Sainsbury

Eiko Saito

Marta Sajdakowska

Carmina Saldana

Silvia Saldiva

Amin Salehi-Abargouei

Rosana Salles-Costa

Marina Salvadori

Rodrigo San Cristobal

Luz María Sánchez-Romero

Emma Sanchez-Vaznaugh

Parvane Saneei

Joseph Santos

Leonardo Santos

Roberta Santos

Lincoln Sargeant

Peter Sarich

Danya Sarkar

Uttam Kumar Sarkar

Flavia Sarti

Daniela Sartorelli

Benn Sartorius

Zvonimir Šatalić

Isabela Sattamini

Mateja Savoie-Roskos

Mathilde Savy

Amy Saxe-Custack

Peter Scarborough

Claudia Schauer

Catarina Schiborn

Rosemary Schleicher

Sabrina Schlesinger

Caroline Schlinkert

Julie Schmidt

Ricarda Schmidt

Christoph Schmoecker

Berta Schnettler

Danielle Schoenaker

Ashley Schram

Joshua Schrock

Jeppe Schroll

Ivonne Schulman
Peter Schulz

Rebecca Schwei

Pietro Scicchitano

Sara Seck

M. Heather Sedibe

Gina Segovia-Siapco

Hilary Seligman

K Selvaraj

Bisakha Sen

Marjanne Senekal

Angan Sengupta

Sara Serahati

Michele R Sgambato

Sakineh Shab-Bidar

Autumn Shafer

Dheeraj Shah

Hossein Shahnazi

Mohd Razif Shahril

Teresa Shamah-Levy

Amy Shanafelt

Kamal Sharma

Shantanu Sharma

David Sharp

Sarah Shaw

Kyla Shea

Shanshan Sheehy

Minxue Shen

Alon Shepon

Zumin Shi

Victoria Shier

Jae Eun Shim

Yoshihiro Shimazaki

Dayeon Shin

Nitin Shivappa

Olayinka Shiyanbola

Sumina Shrestha

Yurii Shvetsov

Rosely Sichieri

Karen Siegel

Madeleine Sigman-Grant

Lastdes Sihombing

Lynn Silver

Anja Simmet

Harbindar Jeet Singh

Rachel Sinley

Susan Sisson

Kara Skelton

Kelly Skinner

Marianne Skreden

Joyce Slater

Alison Smith

Hazel Smith

Michaela Smith

Peter Smyth

Charles Snart

Panmela Soares
Jakub Sobiecki

Damayanti Soekarjo

Golbon Sohrab

Donmozoun Telesphore Some

Yi Song

Sajid Soofi

Katherine Speirs

Naiara Sperandio

Chandrashekhar Sreeramareddy

Chandrashekhar $\mathrm{T}$.

Sreeramareddy

Derrick Ssewanyana

Meir Stampfer

Rosemary Stanton

Nanette Steinle

Steen Stender

Dalia Stern

Robert C. Stewart

Nelia Steyn

Nicole Stoffel

Jodi Stookey

Sofia Strommer

Melissa Strompolis

Amy Subar

Malavika Subramanyam

Christopher Sudfeld

Norhasmah Sulaiman

Carolyn Summerbell

Rachel Sutherland

Carolyn Sutter

Åsa Svensson

Allison Sylvetsky

Garden Tabacchi

Lindsey Smith Taillie

Mohammad Talaei

Alison Talbert

Sameera Talegawkar

Henk Talma

Dessalegn Tamiru Adugna

LiMin Tan-Buchanan

Sherry Tanumihardjo

Xingyong Tao

Valerie Tarasuk

Carla Tarazona-Meza

Adonina Tardón

Marie Tarrant

Carla Taylor

Nathan Taylor

E-Siong Tee

Michelle Teixeira Teixeira

Elisabeth Temme

Carla Teo

Inge Tetens

Chan Thai

Chloe Thomas

Sharon V. Thompson 
PHN List of Reviewers

Janet Thorlton

Anne Thorndike

Andrew Thorne-Lyman

Anne Marie Thow

Changwei Tian

Deirdre Tobias

Ulla Toft

Emily Tomayko

Simone Tomaz

Melody Tondeur

Sandra Torres

Alison Tovar

Pierre Traissac

Gina Trakman

Danielle Treadwell

Indi Trehan

Helen Trevena

Thi Huong Trinh

Ursula Trubswasser

Angela Trude

Caroline Trumpff

A. Tsapanou

Marilyn Tseng

George Tsitsas

Lungiswa Tsolekile

Claire Tugault-Lafleur

Rachel Tumin

Hannah Tuomisto

S. Turner

Jasna Twynstra

Shobha Udipi

Ken Uechi

Mayu Uemura

Mishel Unar-Munguía

Nurhan Unusan

Penney Upton

Avita Usfar

Jennifer Utter

Samuel Uwaezueke

Elisavet Valanou

Desiree Valera-Gran

Maite Vallejo

Louise van den Berg

Ellen van den Heuvel

Jan Willem van der Kamp

Rachel van der Pols-Vijlbrief

Laura van der Velde

Maya Van Gent

Averalda Van Graan

Pat Vanderkooy

Stefanie Vandevijvere

Nadja Vasiljevic

Juliana Vaz
Gabriela Vedovato

Gustavo Velasquez-Melendez

Frederick Veldman

Elizabeth Velema

Kelsey Vercammen

Laura Vergeer

Eric Verger

Eliseu Verly-Jr

Helen Vidgen

Diva Vieira

Reinhold Vieth

Florent Vieux

Frøydis Vik

Mireya Vilar-Compte

Sofia Vilela

Salvador Villalpando

Eduardo Villamor

Michelle Vine

Fernando Vio

Shelina Visram

Marianne Visser

Dorothee Volkert

Rachel Vollmer

Elizabeth Vrany

Kenji Wakai

Lina Walkinshaw

Jordyn Wallenborn

Corinna Walsh

Chongjian Wang

Donqing Wang

Jinwei Wang

Qian Janice Wang

Wendy Watson

Karen Webb

Jacqueline Webster

Hope Weiler

Charles Weitz

Emily Welker

Nancy M. Wells

Jean Welsh

Edelweiss Wentzel-Viljoen

Susanne Westenbrink

Marc White

Hannah White

Zelda White

Susan Whiting

Clare Whitton

Stephen Whybrow

Catherine Wickham

Mariaan Wicks

Elizabeth Widen

Michael Widener

Kurt Widhalm
Frank Wieringa

Hanneke Wijnhoven

Parke Wilde

Emma Wilkins

Sydney Willis

Noreen Willows

Gayle C. Windham

Pattanee Winichagoon

Julia Wolfson

Jyh Eiin Wong

Michelle Wong

Jean Woo

Julie Woods

Kathryn Woods-Townsend

Charlotte Wright

Iwona Wronka

Stephanie Wrottesley

Aozhou Wu

Jason Wu

Qiang Wu

Yangfeng Wu

Amanda Wyatt

Peter Wyman

Rebecca Wyse

Fei Xu

Lin Xu

Xiaoyue Xu

Xijin Xu

Zali Yager

Najat Yahia

Nianhong Yang

Ray-Yu Yang

Xuefeng Yang

Mary Yannakoulia

Amy Yau

Heather Yeatman

Nagarajkumar Yenugadhati

Andy Wai Kan Yeung

Medine Yilmaz

Samrawit Yisahak

Jin-Ha Yoon

Sze Lin Yoong

Candace Young

Sera Young

C.Q. Yu

Elaine $\mathrm{Yu}$

Kang Yu

Zhi Yu

Changzheng Yuan

Luciana Zaccagni

Arwa Zahid

Gerardo Zamora

Raul Zamora-Ros 
Jiali Zeng

Taddese Zerfu

Bin Zhang

Bing Zhang

Cai-Xia Zhang

Jianjun Zhang

Nanhua Zhang
Dong Zhao

Xiang Zhao

Chen Zhen

Ju-sheng Zheng

Zhenghua Zhou

Nida Ziauddeen

Mzondi Ziba
Hana Zickgraf

Deena R. Zimmerman

Armin Zittermann

Stanley Zlotkin

Christina Zorbas

Francis Zotor

Runyu Zou 